\title{
A!
}

This is an electronic reprint of the original article.

This reprint may differ from the original in pagination and typographic detail.

Iyer, Ajai; Etula, Jarkko; Ge, Yanling; Liu, Xuwen; Koskinen, Jari

Nanodiamond embedded ta-C composite film by pulsed filtered vacuum arc deposition from a single target

Published in:

Applied Physics Letters

DOI:

$10.1063 / 1.4967985$

Published: 01/11/2016

Document Version

Publisher's PDF, also known as Version of record

Please cite the original version:

Iyer, A., Etula, J., Ge, Y., Liu, X., \& Koskinen, J. (2016). Nanodiamond embedded ta-C composite film by pulsed filtered vacuum arc deposition from a single target. Applied Physics Letters, 109(20), [201905].

https://doi.org/10.1063/1.4967985

This material is protected by copyright and other intellectual property rights, and duplication or sale of all or part of any of the repository collections is not permitted, except that material may be duplicated by you for your research use or educational purposes in electronic or print form. You must obtain permission for any other use. Electronic or print copies may not be offered, whether for sale or otherwise to anyone who is not an authorised user. 


\section{Nanodiamond embedded ta-C composite film by pulsed filtered vacuum arc deposition from a single target}

Ajai lyer, Jarkko Etula, Yanling Ge, Xuwen Liu, and Jari Koskinen

Citation: Appl. Phys. Lett. 109, 201905 (2016); doi: 10.1063/1.4967985

View online: https://doi.org/10.1063/1.4967985

View Table of Contents: http://aip.scitation.org/toc/apl/109/20

Published by the American Institute of Physics

\section{Articles you may be interested in}

Effect of tetrahedral amorphous carbon coating on the resistivity and wear of single-walled carbon nanotube network

Journal of Applied Physics 119, 185306 (2016); 10.1063/1.4948672

Interfacial $\mathrm{SiO}_{2}$ scavenging kinetics in $\mathrm{HfO}_{2}$ gate stack

Applied Physics Letters 109, 202905 (2016); 10.1063/1.4968002

Reversible magnetocaloric effect in materials with first order phase transitions in cyclic magnetic fields:

$\mathrm{Fe}_{48} \mathrm{Rh}_{52}$ and $\mathrm{Sm}_{0.6} \mathrm{Sr}_{0.4} \mathrm{MnO}_{3}$

Applied Physics Letters 109, 202407 (2016); 10.1063/1.4968241

Photodetection in $\mathrm{p}-\mathrm{n}$ junctions formed by electrolyte-gated transistors of two-dimensional crystals Applied Physics Letters 109, 201107 (2016); 10.1063/1.4967173

Graphene diamond-like carbon films heterostructure

Applied Physics Letters 106, 102108 (2015); 10.1063/1.4914495

Novel phase of carbon, ferromagnetism, and conversion into diamond

Journal of Applied Physics 118, 215303 (2015); 10.1063/1.4936595

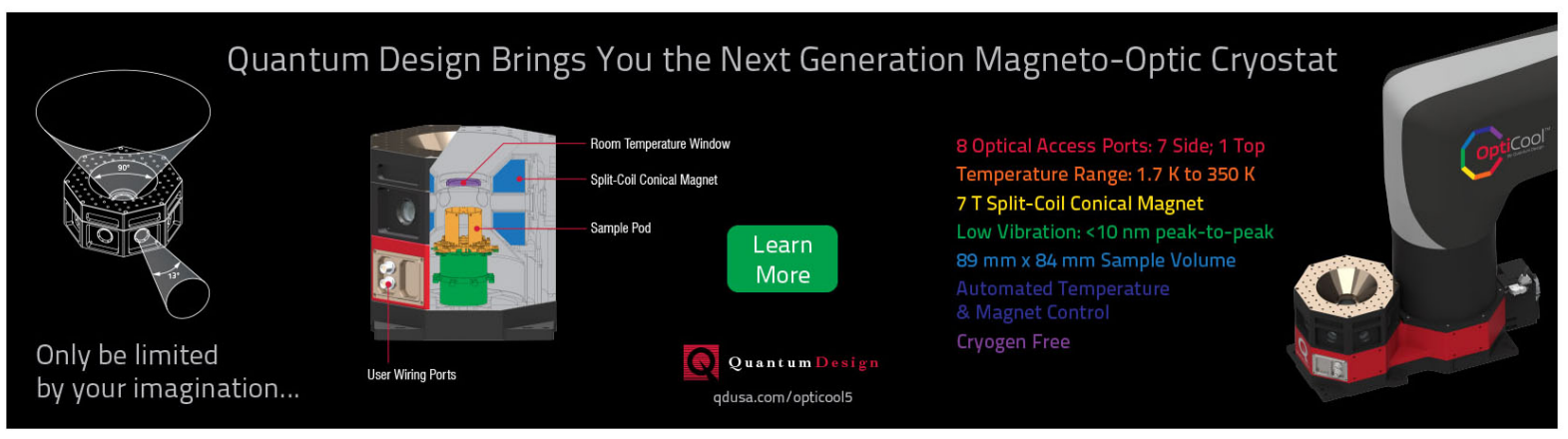




\title{
Nanodiamond embedded ta-C composite film by pulsed filtered vacuum arc deposition from a single target
}

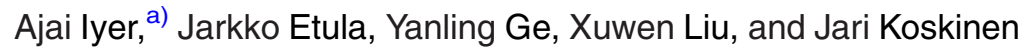 \\ Department of Materials Science and Engineering, School of Chemical Technology, Aalto University, \\ POB 16200, 00076 Espoo, Finland
}

(Received 10 August 2016; accepted 5 November 2016; published online 15 November 2016)

\begin{abstract}
Detonation Nanodiamonds (DNDs) are known to have $\mathrm{sp}^{3}$ core, $\mathrm{sp}^{2}$ shell, small size (few $\mathrm{nm}$ ) and are gaining importance as multi-functional nanoparticles. Diverse methods have been used to form composites, containing detonation nanodiamonds (DNDs) embedded in conductive and dielectric matrices for various applications. Here we show a method, wherein DND-ta-C composite film, consisting of DNDs embedded in ta-C matrix have been co-deposited from the same cathode by pulsed filtered cathodic vacuum arc method. Transmission Electron Microscope analysis of these films revel the presence of DNDs embedded in the matrix of amorphous carbon. Raman spectroscopy indicates that the presence of DNDs does not adversely affect the $\mathrm{sp}^{3}$ content of DND-ta-C composite film compared to ta-C film of same thickness. Nanoindentation and nanowear tests indicate that DND-ta-C composite films possess improved mechanical properties in comparison to ta-C films of similar thickness. Published by AIP Publishing. [http://dx.doi.org/10.1063/1.4967985]
\end{abstract}

Nanodiamonds (NDs) have found wide fields of application due to the presence of stable diamond core, leading to versatile properties. ${ }^{1}$ However, growth of nanodiamonds on any surface is generally achieved by chemical vapour deposition (CVD) at elevated temperatures. ${ }^{2-5}$ In many cases, such high temperature processes cannot be performed due to substrate considerations or in cases where the NDs need to be embedded in a matrix. Thus, it is easier to proceed from pre-made NDs, such as those synthesized by a detonation process. ${ }^{1}$ Detonation nanodiamonds (DNDs) have a diamond core surrounded by $\mathrm{sp}^{2}$ carbon shell which can easily be functionalized for a variety of applications. ${ }^{1}$ It has been shown that incorporation of DNDs in an epoxy matrix ${ }^{6,7}$ or metal matrix ${ }^{8,9}$ can result in improved mechanical properties of the composite. However, making any hard, thin film coating with DNDs alone, without embedding them in a matrix material, is impossible.

Diamond like Carbon (DLC) is an amorphous carbon coating which is very hard and is used as a mechanical coating at an industrial scale, with Tetrahedral Amorphous Carbon (ta-C) being the best possible DLC coating. ${ }^{10}$ DLC/ ta-C films with NDs have been fabricated either by depositing the NDs before DLC/ta-C film growth ${ }^{11}$ or by CVD methods at high temperature, where process parameters must be varied to cater for nucleation of NDs and DLC separately. ${ }^{3,12}$

In this publication, we present a method of codepositing DNDs and ta-C from the same cathode using a pulsed filtered cathodic vacuum arc process ( $\mathrm{p}-\mathrm{FCVA}$ ) at ambient temperatures. In essence, the carbon cathode was loaded with DNDs some of which were ejected in the process of carbon arc plasma formation. Without going into the details of cathodic $\operatorname{arcs}^{13}$ at the arc spot, carbon cathode gets converted into plasma with nano and micro particles which was directed to the substrate through a macro-particle filter.

\footnotetext{
${ }^{\text {a) }}$ Author to whom correspondence should be addressed. Electronic mail: ajai.iyer@aalto.fi. Tel.: +358504603256.
}

The ejected DNDs travel in the carbon plasma stream and are not filtered by the macro-particle filter if they are few tens to few hundreds of $\mathrm{nm}$ in size. ${ }^{13}$ On the substrate, the DNDs get embedded in the growing ta-C film forming a nano-composite film by a one-step deposition process operating at room temperature.

The deposition system consists of a vacuum chamber which can be evacuated to pressures lower than $2 \times 10^{-7}$ Torr $\left(2.66 \times 10^{-5} \mathrm{~Pa}\right)$. The p-FCVA system uses a $45^{\circ}$ bent filter, with a pulse forming network charged to $-400 \mathrm{~V}$ (pulse current $650 \mathrm{~A}$ ) to initiate the arc between the cathode and anode. The triggerless $\operatorname{arc}^{14}$ was initiated in pulses, at a repetition frequency of $1.0 \mathrm{~Hz}$, by use of a self-designed Labview based software, controlling National Instruments (NI) hardware.

Graphite rods of diameter $6.35 \mathrm{~mm}$ and purity of 99.995\% (Graphitestore) were used as cathodes and charged with DNDs. The DNDs in stable water suspension $\left(\right.$ Andante ${ }^{\mathrm{TM}}$ ) with $5 \mathrm{wt}$. \% of DNDs and average single digit size of $5 \mathrm{~nm}$ (Carbodeon Ltd) was used to charge the cathodes. The carbon cathode was drilled with 7 holes (hole diameter $1.5 \mathrm{~mm}$ and hole depth $3 \mathrm{~mm}$ ) distributed over the cathode area. $100 \mu \mathrm{l}$ of DND solution was drop cast into the holes following which the graphite rod was dried on a hot plate at $200{ }^{\circ} \mathrm{C}$ for $120 \mathrm{~min}$. After the $120 \mathrm{~min}$ drying time, the graphite rod was allowed to cool, and the charging procedure was repeated once more.

Intrinsic silicon (100) cut into $3 \mathrm{~cm} \times 2 \mathrm{~cm}$ was used as substrates. The substrates were ultrasonically cleaned in high purity liquid chromatography (HPLC) grade acetone and blow dried by $\mathrm{N}_{2}$ gas. For TEM analysis, salt crystals (Tedpella) cleaved into $1 \mathrm{~cm} \times 1 \mathrm{~cm}$ pieces and ultrasonically cleaned by HPLC grade acetone were used as substrates. Post deposition, the salt crystals were put as the film sides up in a shallow bath of de-ionized water until the film delaminates. The delaminated film was fished out onto M75 copper only TEM grids (Agar) and used for TEM analysis. For comparative TEM analysis original DND solution was 
diluted to $0.05 \mathrm{wt}$. \% and drop cast onto 400 mesh copper grids with holey carbon film (Agar). Film thickness of $50 \mathrm{~nm}$ (500 pulses) was deposited for both ta-C and DND-ta-C for comparative analysis. The substrate was placed $110 \mathrm{~mm}$ away from the plasma filter coil at floating potential and room temperature. The substrate was rotated at $17 \mathrm{rpm}$ to ensure uniform film thickness over the whole area. The deposition rate for both ta-C and DND-ta-C composite film was found to be around $0.1 \mathrm{~nm} /$ pulse for $110 \mathrm{~mm}$ substrate to filter coil distance by checking the film thickness using a contact profilometer (Dektak 6M).

TEM analysis was performed by a Tecnai F-20 (FEI) TEM operating at $200 \mathrm{kV}$ and acquired images were processed by Digital Micrograph software (Gatan). SEM analysis of the samples was performed by a Hitachi S-4700 SEM. Raman spectroscopy was performed by a Labram HR (Horiba Jobin-Yvon) confocal Raman system (Ar laser $\lambda=488 \mathrm{~nm}$ and power $=10 \mathrm{~mW}$ ) using $100 \times$ objective
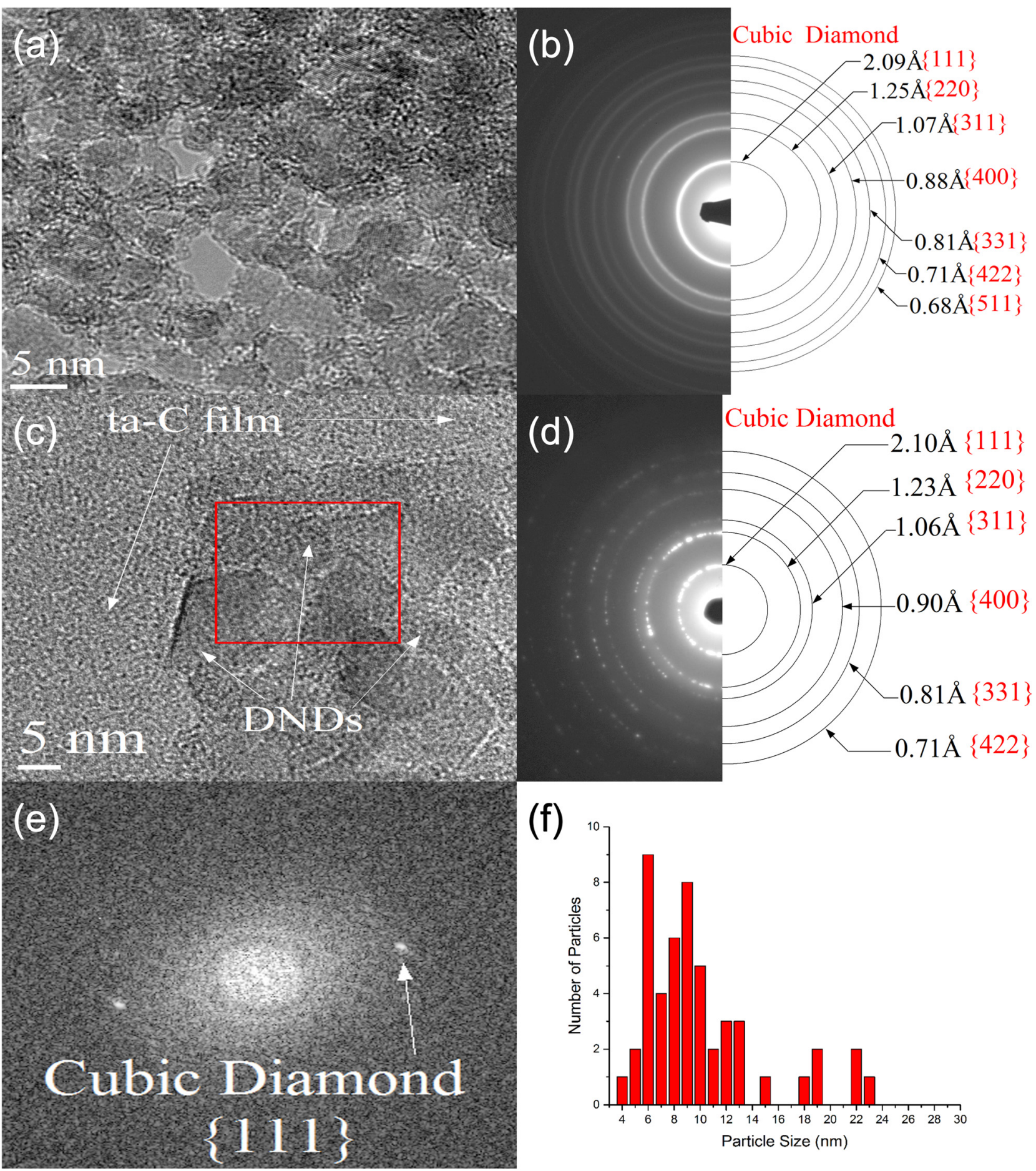

(f)

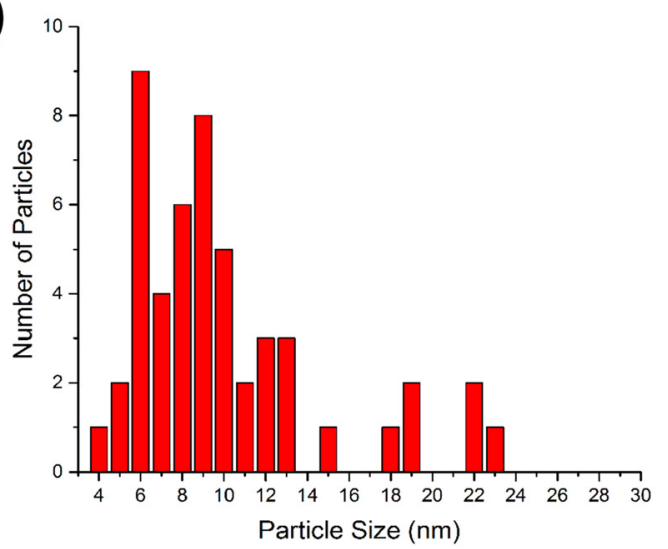

FIG. 1. HRTEM images and SAED ring patterns of samples. (a) HRTEM image of original DND solution; (b) SAED ring pattern of original DND solution indexed to cubic diamond phase (marked in red); (c) HRTEM image of DND-ta-C composite film. The DND particles are marked. The amorphous film in the image is ta-C; (d) SAED ring pattern of DND-ta-C composite film from DND agglomerate indexed to cubic diamond phase (marked in red); (e) FFT of the area indicated by red square in (c). The cubic diamond phase is marked; (f) Particle size histogram for 50 particles from HRTEM image analysis. 
TABLE I. Spacing (d) measured by SAED in this work indexed to d-spacing of cubic diamond (Fd3m with $\mathrm{a}_{0}=3.56 \AA$ ) from literature. ${ }^{15-17}$

\begin{tabular}{lcc}
\hline \hline Cubic Diamond $^{15-17} \mathrm{~d}(\AA)\{\mathrm{hkl}\}$ & Original DND solution $\mathrm{d}_{\text {measured }}(\AA)$ & DND-ta-C composite $\mathrm{d}_{\text {measured }}(\AA)$ \\
\hline $2.06\{111\}$ & 2.09 & 2.10 \\
$1.26\{220\}$ & 1.25 & 1.23 \\
$1.07\{311\}$ & 1.07 & 1.06 \\
$0.89\{400\}$ & 0.88 & 0.90 \\
$0.81\{331\}$ & 0.81 & 0.81 \\
$0.72\{422\}$ & 0.71 & 0.71 \\
$0.68\{511\}$ & 0.68 & $\ldots$ \\
\hline \hline
\end{tabular}

(Olympus) with a spot size of around $1 \mu \mathrm{m}$ and aperture size of $1000 \mu \mathrm{m}$ to maximize the signal from DNDs.

Nano wear and Nano indentation tests were performed using a TI-900 (Hysitron Inc.) system using a Berkowitz tip (nominal diameter $200 \mathrm{~nm}$ ). For both the DND-ta-C and reference ta-C films, to reduce contribution of the silicon substrate, the nano-indentation testing was performed in two stages. In the first stage, 10 point indentation was performed to estimate the optimal depth from load-displacement curves. In the second stage, an untouched area was selected and 12 points were indented in a displacement controlled manner set for the optimal depth as found from the previous stage. Wear tests were performed at 4 points with loads of $50 \mu \mathrm{N}$ to $200 \mu \mathrm{N}$ with increments of $50 \mu \mathrm{N}$ for every point. Each wear point scan area was $1 \mu \mathrm{m} \times 1 \mu \mathrm{m}$, and tests were performed with 2 and 4 wear scan passes to compare the wear with increase in number of scan passes. Post wear images were acquired by using a small force of $1.5 \mu \mathrm{N}$, and the image area was $3 \mu \mathrm{m} \times 3 \mu \mathrm{m}$. The wear images were analysed by Gwyddion and the average crater wear volume was calculated as a product of the average crater wear depth and the average crater area for each load. The wear volume ratio was calculated as the ratio of the average crater wear volume to the maximum possible wear volume (film thickness $\times 1 \mu \mathrm{m}^{2}$ ).

Cross-sectional SEM images (not shown) of the DNDta-C film, indicate the film thickness to be around $55 \pm 3 \mathrm{~nm}$ which compares well with the expected film thickness of $50 \mathrm{~nm}$.

The representative HRTEM image and selected area electron diffraction (SAED) ring pattern from the original DND solution drop cast onto the TEM grid are shown in Figs. 1(a) and 1(b). From the SAED ring pattern of the original DNDs (indexed in Fig. 1(b) and Table I), the primary d-spacings were found to be $2.09 \AA, 1.25 \AA$, and $1.07 \AA$, which matches closely with values in literature for cubic diamond nanoparticles. ${ }^{15-17}$ Representative HRTEM image of the DND-ta-C composite film (Fig. 1(c)) indicates DND agglomerate embedded (marked in image) in a matrix of amorphous ta-C. The SAED ring pattern from a DND agglomerate in the DND-ta-C composite film (indexed in Fig. 1(d) and tabulated in Table I) indicates a cubic diamond phase. The cubic diamond phase can also be observed from the FFT of HRTEM image as shown in Fig. 1(e). It must be pointed out that the SAED ring patterns from the majority of the composite film area consist of partial rings with a weak intensity, indicating the presence of DNDs. This is attributed to the extremely small size (average diameter of $5 \mathrm{~nm}$ ) and low concentration of DNDs in the composite film. Complete SAED ring patterns are observed with larger DND agglomerate (a few tens of $\mathrm{nm}$ ) as shown in Fig. 1(d). Similarly stable and high quality HRTEM images could be obtained only for larger DND agglomerates while individual DNDs were graphitized in a short time when exposed to the electron beam. ${ }^{18}$ The graphitization time and extent is dependent on the size of the DNDs and the smaller DNDs graphitize faster than the large agglomerates. ${ }^{18}$ The SAED ring patterns from reference ta-C film (not shown) are typical of amorphous films and show no crystallinity whatsoever. Energy dispersive $\mathrm{X}$-ray spectroscopy (EDX) performed using a small electron beam probe on a large number of particles in the DND-ta-C film (results not shown here) indicated only carbon $\mathrm{K} \alpha$ line as the relevant peak in the spectrum. Particle analysis from the
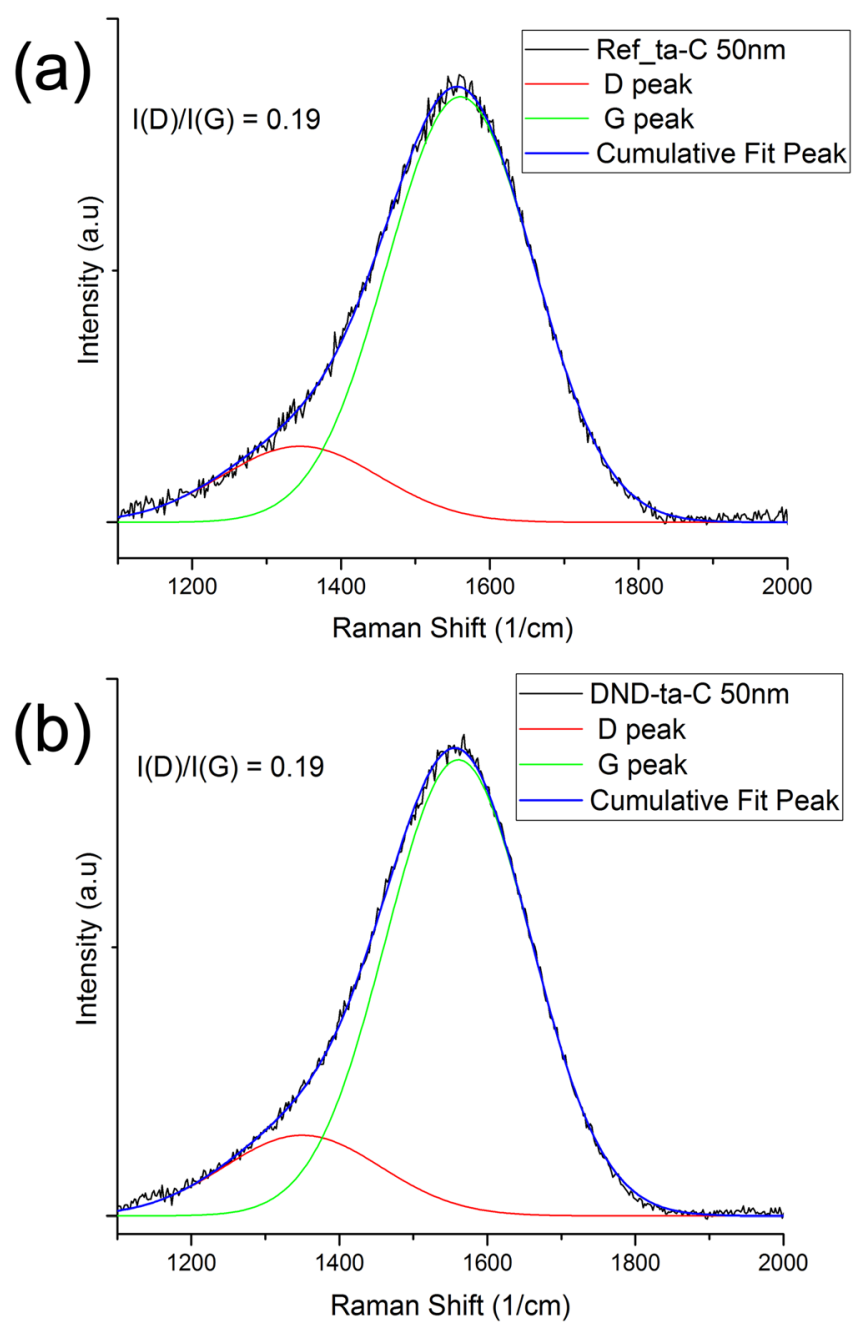

FIG. 2. Raman spectra acquired at $488 \mathrm{~nm}$ wavelength. (a) Reference ta-C $50 \mathrm{~nm}$ thick; (b) DND-ta-C $50 \mathrm{~nm}$ thick. 

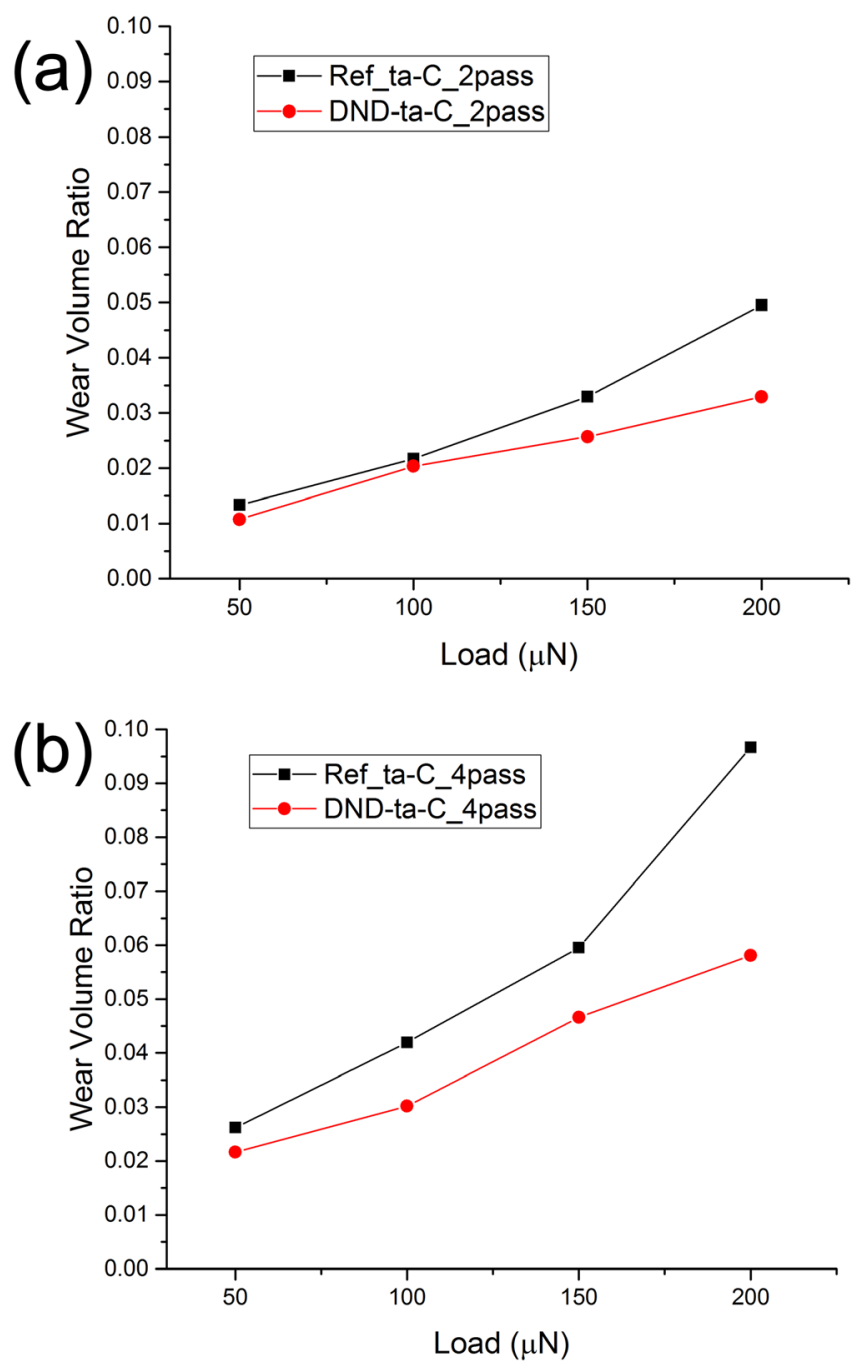

FIG. 3. Nano-wear plots. (a) Wear volume ratio for reference ta-C film and DND-ta-C film as a function of load plotted for 2 scan passes; (b) Wear volume ratio for reference ta-C film and DND-ta-C film as a function of load plotted for 4 scan passes.

HRTEM images indicates an average of around 17000 DNDs per $\mu \mathrm{m}^{3}$, dispersed as small agglomerates (Fig. 1(f)). From the particle analysis, the DND concentration was calculated to be on the order of $0.1 \mathrm{vol} . \%$ in the DND-ta-C film.

Raman spectra for both reference ta-C and DND-ta-C film were acquired and fitted by two peaks for D and G regions of amorphous carbon as per literature ${ }^{10,19}$ and representative plots are shown in Fig. 2. The $\mathrm{I}(\mathrm{D}) / \mathrm{I}(\mathrm{G})$ ratios for both the $50 \mathrm{~nm}$ thick films were calculated from 3 random points each and averaged. The $\mathrm{I}(\mathrm{D}) / \mathrm{I}(\mathrm{G})$ ratios show no substantial difference between reference ta-C and DND-ta-C film, indicating that the $\mathrm{sp}^{3}$ ratio of the films are unaffected by the DNDs. Note that the DND peaks (expected around $1325-1332 \mathrm{~cm}^{-1}$ ) are not visible in the Raman spectra, most probably due to the low concentration of DNDs in the ta-C matrix (as shown above) and heavy attenuation of the $\mathrm{sp}^{3}$ signal due to $\mathrm{sp}^{2}$ content in the ta-C film. ${ }^{10,19}$

The nano-indentation data was analysed by Hysitron Inc. software using Oliver-Pharr method. ${ }^{20}$ The tip area function was calibrated before and after the indentation and wear tests. For both samples the average value of hardness was calculated by averaging the 12 point hardness data and further averaged for tip area function. The average hardness for reference ta-C film is around $30 \mathrm{GPa}$, which matches with the data in Refs. 10 and 21 while for the DND-ta-C film, the average hardness is around $35 \mathrm{GPa}$. The wear volume ratio plots for 2 and 4 wear scans are shown in Figs. 3(a) and 3(b), respectively. It can be observed from the wear volume ratio plots (Fig. 3) that the wear volume ratio of reference ta-C film increases sharply as the load and number of passes increase while, the DND-ta-C film wear volume ratio has only a modest increase over the whole range of loads and passes. From the DND concentration, we estimate that there would be few tens of DNDs below the indenter tip at any given position and time. The presence of embedded DNDs appears to improve the mechanical properties of the DND-ta-C film as the nanoindentation data indicates that the DND-ta-C is harder and more wear resistant than reference ta-C.

In summary, we have shown a method of co-depositing DND embedded ta-C composite thin films by a single step, room temperature p-FCVA process. The TEM analysis of DND-ta-C composite film indicates the presence of DNDs embedded in the ta-C matrix. The small quantity of DNDs (on the order of $0.1 \mathrm{vol} . \%$ ) in the DND-ta-C composite film, seems to have no effect on the $\mathrm{sp}^{3}$ ratio of the film, but the composite film is observed to be harder and more wear resistant than ta-C film of the same thickness. Also cyclic voltammetry tests indicate that the already fabricated DND-ta-C composite film is a very sensitive bio-sensor film, and further tests are in progress.

The support given by the Academy of Finland through project HISCON (No. 259595) is gratefully acknowledged.

The help of Mr. Niklas Wester for cyclic voltammetry tests is gratefully acknowledged.

${ }^{1}$ V. N. Mochalin, O. Shenderova, D. Ho, and Y. Gogotsi, "The properties and applications of nanodiamonds," Nat. Nanotechnol. 7, 11 (2012).

${ }^{2}$ N. Jiang, S. Kujime, I. Ota, T. Inaoka, Y. Shintani, H. Makita, A. Hatta, and A. Hiraki, "Growth and structural analysis of nano-diamond films deposited on $\mathrm{Si}$ substrates pretreated by various methods," J. Cryst. Growth 218, 265 (2000).

${ }^{3}$ N. Sakudo, N. Ikenaga, H. Yasui, and K. Awazu, "Amorphous carbon coating mixed with nano-diamonds," Thin Solid Films 516, 4483 (2008).

${ }^{4} \mathrm{Q}$. Chen, J. Yang, and Z. Lin, "Synthesis of oriented textured diamond films on silicon via hot filament chemical vapor deposition," Appl. Phys. Lett. 67, 1853 (1995).

${ }^{5}$ D. Varshney, M. Ahmadi, M. J.-F. Guinel, B. R. Weiner, and G. Morell, "Single-step route to diamond-nanotube Composite," Nanoscale Res. Lett. 7, 535 (2012).

${ }^{6}$ I. Neitzel, V. Mochalin, I. Knoke, G. R. Palmese, and Y. Gogotsi, "Mechanical properties of epoxy composites with high contents of nanodiamond," Compos. Sci. Technol. 71, 710 (2011).

${ }^{7}$ Y.-J. Zhai, Z.-C. Wang, W. Huang, J.-J. Huang, Y.-Y. Wang, and Y.-Q. Zhao, "Improved mechanical properties of epoxy reinforced by low content nanodiamond powder," Mater. Sci. Eng., A 528, 7295 (2011).

${ }^{8}$ I. M. Melendez, E. Neubauer, P. Angerer, H. Danninger, and J. M. Torralba, "Influence of nano-reinforcements on the mechanical properties and microstructure of titanium matrix composites," Compos. Sci. Technol. 71, 1154 (2011).

${ }^{9}$ K. Hanada, K. Yamamoto, T. Taguchi, E. Osawa, M. Inakuma, V. Livramento, J. B. Correia, and N. Shohoji, "Further studies on copper nanocomposite with dispersed single-digit-nanodiamond particles," Diamond Relat. Mater. 16, 2054 (2007).

${ }^{10}$ J. Robertson, "Diamond like amorphous carbon," Mater. Sci. Eng. R 37, 129 (2002). 
${ }^{11}$ W. G. Xie, J. Chen, W. W. Ming, J. Chen, J. Zhou, S. Z. Deng, and N. S. $\mathrm{Xu}$, "Preparation and field emission property of nanodiamond-clusterembedded diamondlike carbon film," J. Vac. Sci. Technol., B 26, 1321 (2008).

${ }^{12}$ W. Kulisch, C. Popov, S. Boycheva, L. Buforn, G. Favaro, and N. Conte, "Mechanical properties of nanocrystalline diamond/amorphous carbon composite films prepared by microwave plasma chemical vapour deposition," Diamond Relat. Mater. 13, 1997 (2004).

${ }^{13}$ A. Anders, Cathodic Arcs: From Fractal Spots to Energetic Condensation, Springer Series on Atomic, Optical and Plasma Physics No. 50 (Springer, 2008).

${ }^{14}$ A. Anders, I. G. Brown, R. A. MacGill, and M. R. Dickinson, “'Triggerless' triggering of vacuum arcs,” J. Phys. D: Appl. Phys. 31, 584 (1998).

${ }^{15}$ A. Kumar, P. A. Lin, A. Xue, B. Hao, Y. K. Yap, and R. M. Sankaran, "Formation of nanodiamonds at near-ambient conditions via microplasma dissociation of ethanol vapour," Nat. Commun. 4, 2618 (2014).
${ }^{16} \mathrm{P}$. Nemeth, L. A. J. Garvie, and P. R. Buseck, "Twinning of cubic diamond explains reported nanodiamond polymorphs," Sci. Rep. 5, 18381 (2015).

${ }^{17} \mathrm{H}$. Hirai and K. Kondo, "Modified phases of diamond formed under shock compression and rapid quenching," Science 253, 772 (1991).

${ }^{18}$ J. Hiraki, H. Mori, E. Taguchi, H. Yasuda, H. Kinoshita, and N. Ohmae, "Transformation of diamond nanoparticles into onion-like carbon by electron irradiation studied directly inside an ultrahigh-vacuum transmission electron microscope," Appl. Phys. Lett. 86, 223101 (2005).

${ }^{19}$ A. C. Ferrari and J. Robertson, "Raman Spectroscopy of amorphous, nanostructures, diamond-like carbon and nanodiamond," Philos. Trans. R. Soc. London, A 362, 2477 (2004).

${ }^{20} \mathrm{~W}$. C. Oliver and G. M. Pharr, "Measurement of hardness and elastic modulus by instrumented indentation: Advances in understanding and refinements to methodology," J. Mater. Res. 19, 3 (2004).

${ }^{21}$ P. Lemoine, J. P. Quinn, P. Maguire, and J. A. McLaughlin, "Comparing hardness and wear data for tetrahedral amorphous carbon and hydrogenated amorphous carbon thin films," Wear 257, 509 (2004). 